\title{
Die Bedeutung der Tintinnen als Glied der Nahrungskette ${ }^{1}$
}

\author{
BernT ZeITZSCHEL \\ Institut für Meereskunde, Kiel
}

\begin{abstract}
The significance of the tintinnids as a link in the food web. Tintinnids are shell building Protozoa regarded as heterotrich ciliates. Most of them are marine; less than $2 \%$ of the 800 known species occur in fresh water. Tintinnids live almost exclusively free-swimming pelagic and occur at all latitudes, in all seas, predominantly in the upper illuminated water layers. Their geographical distribution is primarily controlled by temperature and so is their development; salinity and oxygen seem of little importance. Tintinnids are one of the first links in the food chain. They feed on detritus, bacteria, naked flagellates, coccolithophores, peridineans and diatoms. The tintinnids themselves are eaten by copepods, euphausiids, tunicates and fish larvae.
\end{abstract}

\section{ALLGEMEINE CHARAKTERISTIK DER TINTINNEN}

Die ersten Angaben über Tintinnen finden sich in einer Arbeit von Müller aus dem Jahre 1776. Seither haben sich zahlreiche Forscher mit dieser Protozoengruppe beschäftigt; besonders hervorzuheben sind die Arbeiten von Clapark̀de \& LaChManN (1858), Daday (1887), Brandt (1906, 1907), Entz (1909), Fauré-Fremiet (1924), Jørgensen (1924, 1927) sowie Kofold \& Campbell (1929, 1939). Tintinnen sind gehäusebildende Protozoen, die etwa $40 \%$ der Ciliaten ausmachen. Bisher sind etwa 800 Arten beschrieben worden; diese sind bis auf einige Ausnahmen marin.

Abbildung 1 gibt einen Überblick über die Organisation der Tintinnen. Der trompetenförmige Weichkörper läuft in einen kontraktilen Stiel aus, mit dem er am Gehäuse befestigt ist. Das Gehäuse, die sogenannte Lorica, besteht aus körpereigener Substanz. CAMPBELI (1954) bezeichnete sie als resistenten, komplexen, organischen Stoff, in den häufig Fremdpartikel eingebaut werden. Die Länge der Lorica liegt bei den rezenten Formen zwischen $20 \mu$ und $1500 \mu$. Der Weichkörper der Tintinnen besitzt meist je 2 Makro- und Mikronuklei. Am oralen Ende ist ein rundes Peristom ausgebildet, welches das Cytostom umgibt. Das Peristom ist von 12 bis $24 \mathrm{kranzförmigr}$ angeordneten Membranellen gesäumt (vgl. Abb. 2).

Die Fortpflanzung dieser Ciliaten ist noch recht ungenügend bekannt. Zweiteilung

1) Diese Untersuchungen wurden zum Teil gefördert durch den Kontrakt N 62 558-3612 zwischen Office of Naval Research, Department of the United States Navy und der Scottish Marine Biological Association. 
scheint vorzuherrschen, doch sind von SCHWEYER (1909) auch Sprossungsvorgänge beobachtet worden; daß Konjugation stattfindet, wird vermutet, ist aber bisher nicht eindeutig nachgewiesen. Dauerzysten können bei ungünstigen Bedingungen gebildet werden. Die Gestalt und die Struktur der Lorica sind die wichtigsten systematischen Merkmale, da meistens der Weichkörper in Netzfängen nicht mehr enthalten oder durch das Fixieren stark beschädigt ist. Das Längenwachstum der Lorica wird durch die

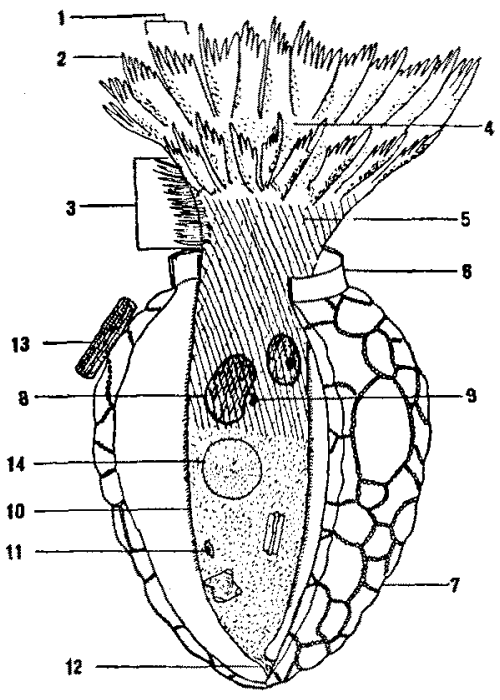

Abb. 1

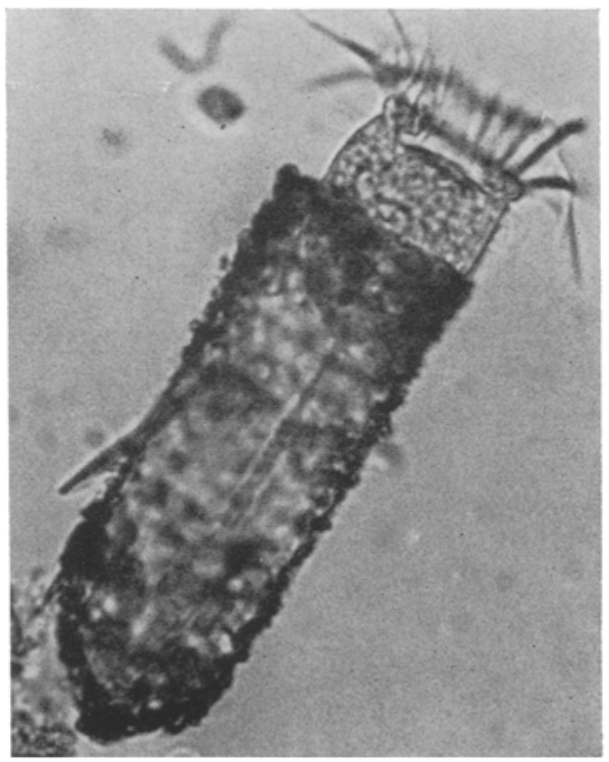

Abb. 2

Abb. 1: Schematische Ubersicht der Organisation von Tintinnen (nach GökE 1963, verändert nach Camprell 1954). Stenosemella nivalis Meunier (Pazifik) mit geöffneter Lorica. 1 Membranellen, 2 Lamina, 3 Ciliarmembran, 4 Lage des Peristoms, 5 Ciliarlinien, 6 Kragen der Lorica, 7 Lorica, 8 Makronukleus, 9 Mikronukleus, 10 Cilien, 11 Analpore, 12 Fuß, 13 agglutinierte Diatomee, 14 Nahrung (Diatomee)

Abb. 2: Tintinnopsis tubulosa LeVANDER, Länge der Lorica etwa $100 \mu$ (9.7.66 Kieler Förde)

Wassertemperatur beeinflußt und ist daher kein eindeutiges systematisches Merkmal. Das Verhältnis der Länge zur oralen Öffnung der Hülse ist jedoch bei vielen Arten konstant und wird deshalb zur Bestimmung herangezogen. Die Lorica schützt zum einen den Weichkörper, hat zum anderen aber vor allen Dingen die Aufgabe, die Fortbewegung zu erleichtern und das Absinken zu verlangsamen. Beim Schwimmen ist das orale Ende meist nach hinten gerichtet, die Lorica dreht sich dabei schraubenförmig durchs Wasser.

\section{DER EINFLUSS VON UMWELTFAKTOREN AUF DIE VERBREITUNG}

Für das Verständnis der Bedeutung der Tintinnen in der Nahrungskette sind folgende Faktoren wichtig: die regionale, vertikale und jahreszeitliche Verbreitung, 

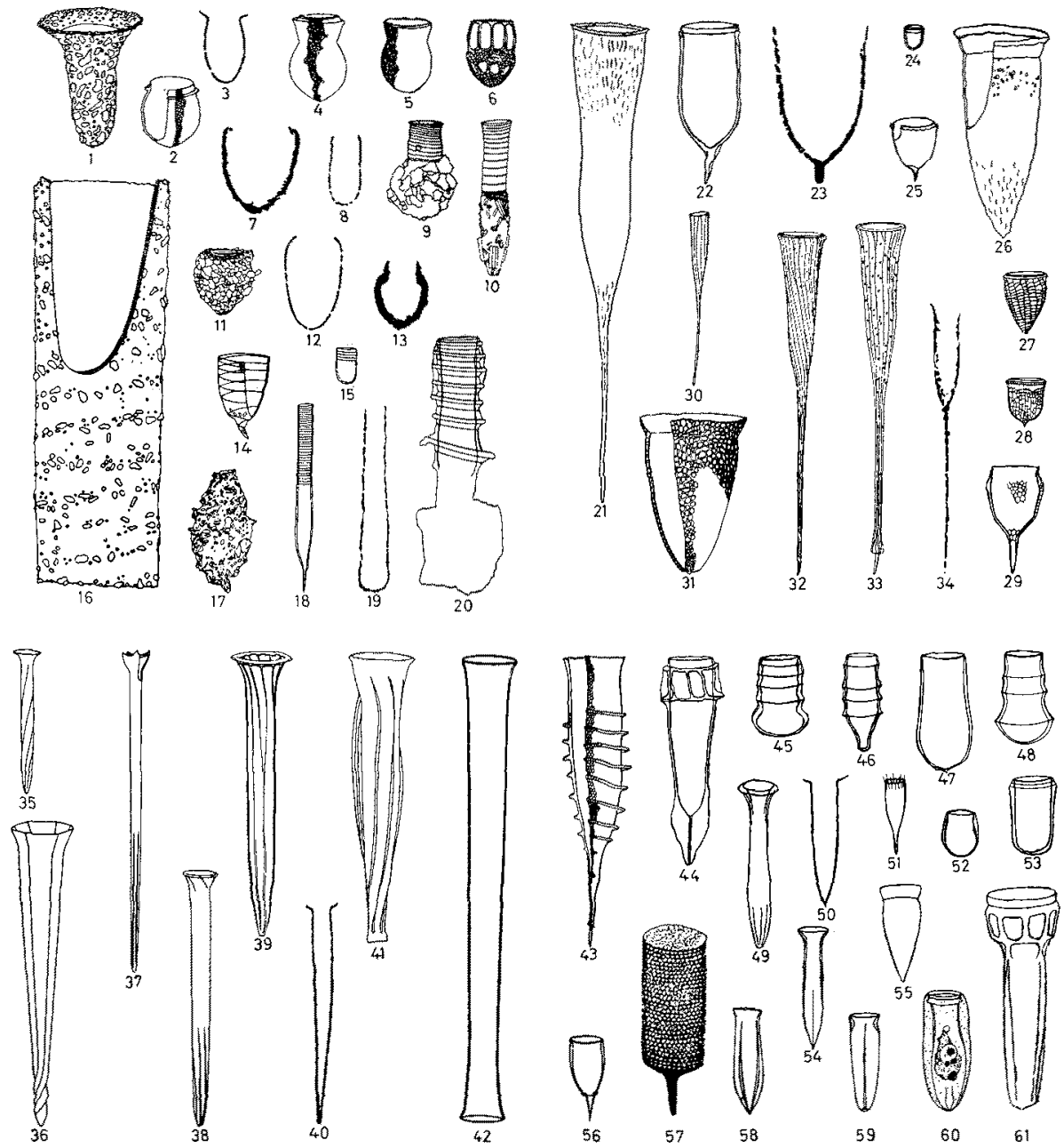

Abb. 3: Obersicht der Tintinnen-Gattungen (nach Campuell 1954). 1 Tintinnopsis, 2 Codonopsis, 3 Tintinnopsella, 4 Codonaria, 5 Codonella, 6 Dictyocysta, 7 Calpionellites, 8 Calpionellopsis, 9 Codonellopsis, 10 Laackmanniella, 11 Stenosemella, 12 Stenosemellopsis, 13 Calpionella, 14 Coxliellat, 15 Metacyclis, 16 Leprotintinnus, 17 Tintinnidium, 18 Helicostomella, 19 Coxliellina, 20 Climacocylis, 21 Cymatocyclis, 22 Favella, 23 Favelloides, 24 Craterella, 25 Acanthostomella, 26 Petalotricha, 27 Epicancella, 28 Epiorella, 29 Epiplocylis, 30 Protorrbabdonella, 31 Cyttarocylis, 32 Rhabdonella, 33 Rhabdonellopsis, 34 Rhabdonelloides, 35 Salpingelloides, 36 Rhabdosella, 37 Salpingacantha, 38 Salpingella, 39 Epicranella, 40 Salpingellina, 41 Daturella, 42 Eutintinnus, 43 Xystonella, 44 Xystonellopsis, 45 Amplectellopsis, 46 Cricundella, 47 Undella, 48 Amplectella, 49 Steenstrupiella, 50 Amphorellina, 51 Dadayiella, 52 Proplectella, 53 Undellopsis, 54 Amphorellopsis, 55 Ormosella, 56 Parundella, 57 Parafavella, 58 Odontophorella, 59 Amphorella, 60 Brandtiella, 61 Stelidiella

sowie der Einfluß von Umweltfaktoren wie $\mathrm{t}^{0}, \mathrm{~S} \%, \mathrm{O}_{2}$ und $\mathrm{pH}$ auf die Entwicklung von Tintinnen. Im folgenden möchte ich kurz auf diese Punkte eingehen.

Viele Tintinnen sind kosmopolitisch verbreitet, und Gebiete des Weltmeeres mit 
ähnlichen Lebensbedingungen beherbergen gleiche'Tintinnenarten. Die Fauna des Nordpazifik ähnelt der des Nordatlantik, die der pazifischen Aquatorregion der des atlantischen Gebietes am Äquator. Die Tintinnengattungen der Arktis und Antarktis sind jedoch vollkommen verschieden. Neben ozeanischen Arten, die meist durchscheinende,
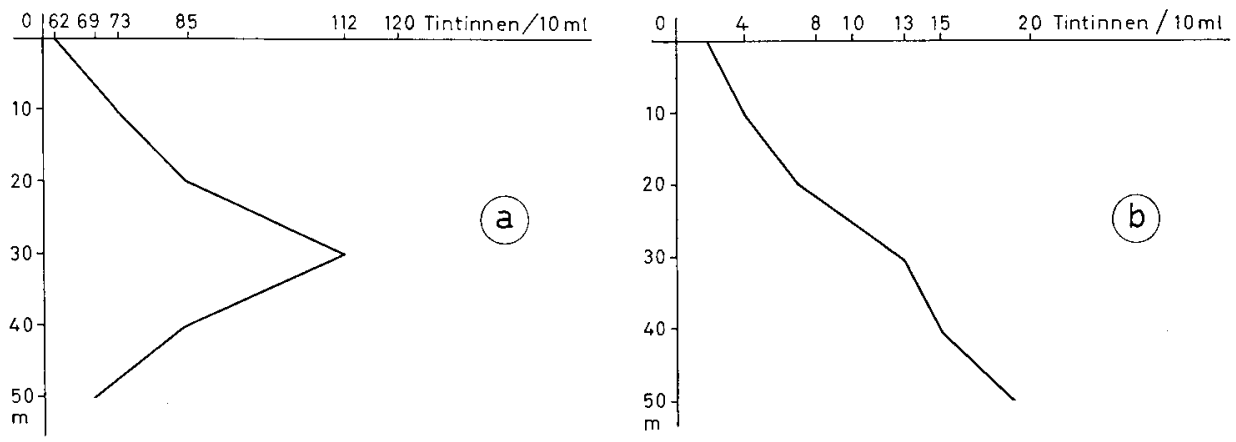

Abb. 4: Vertikale Verteilung von Tintinnen in der Bucht von Alger (nach Vitiello 1964), (a) in der Zeit von November 1962 bis Mai 1963, (b) im Mai 1963
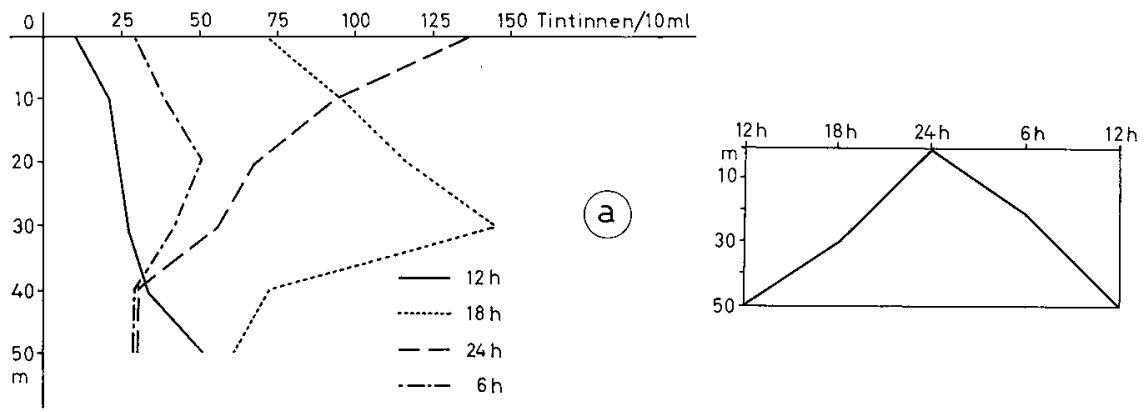

Abb. 5: Vertikale Verteilung von Tintinnen in der Bucht von Alger (nach Vitreldo 1964), (a) Verschiebung der Maxima innerhalb von 24 Std, (b) Schema der Vertikalwanderung innerhalb 24 Std

empfindliche Hülsen besitzen, gibt es einige Gattungen mit gedrungenen, dickwandigen Gehäusen, wie zum Beispiel Tintinnopsis und Stenosemella, die vorwiegend neritisch vorkommen. Sehr auffällig ist, daß große und sehr große Gehäuse in den polaren Meeren häufig sind, während kleine, mittlere und nur wenige große Formen in den tropischen Meeren zu finden sind. Abbildung 3 vermittelt eine Ubersicht von Gattungen der Tintinnen (nach Campbell 1954).

Die vertikale Verteilung von Tintinnen im Mittelmeer ist von VItrello (1964) untersucht worden. Abbildung 4 zeigt, daß das Maximum des Vorkommens in der Bucht von Alger in der Zeit zwischen November und Mai in $30 \mathrm{~m}$ Tiefe liegt, während im Mai das Hauptvorkommen bei $50 \mathrm{~m}$ anzutreffen ist. Abbildung 5 verdeutlicht, $\mathrm{daß}$ eine tägliche Wanderung bei Tintinnen zu beobachten ist. Um 12 Uhr liegt das 
Maximum in $50 \mathrm{~m}$, um 18 Uhr bei $30 \mathrm{~m}$, während es um Mitternacht an der Wasseroberfläche eintritt.

Gillbricht (1954) untersuchte den Einfluß des Lichtes und der Sprungschicht auf die vertikale Verbreitung von Tintinnen. Er kam zu dem Schluß, daß die Vertikalverbreitung dieser Ciliaten vom Licht und von Sprungschichten bestimmt ist. Bei den Sprungschichten kann es sich um thermische oder haline handeln; nicht Absolutwerte,

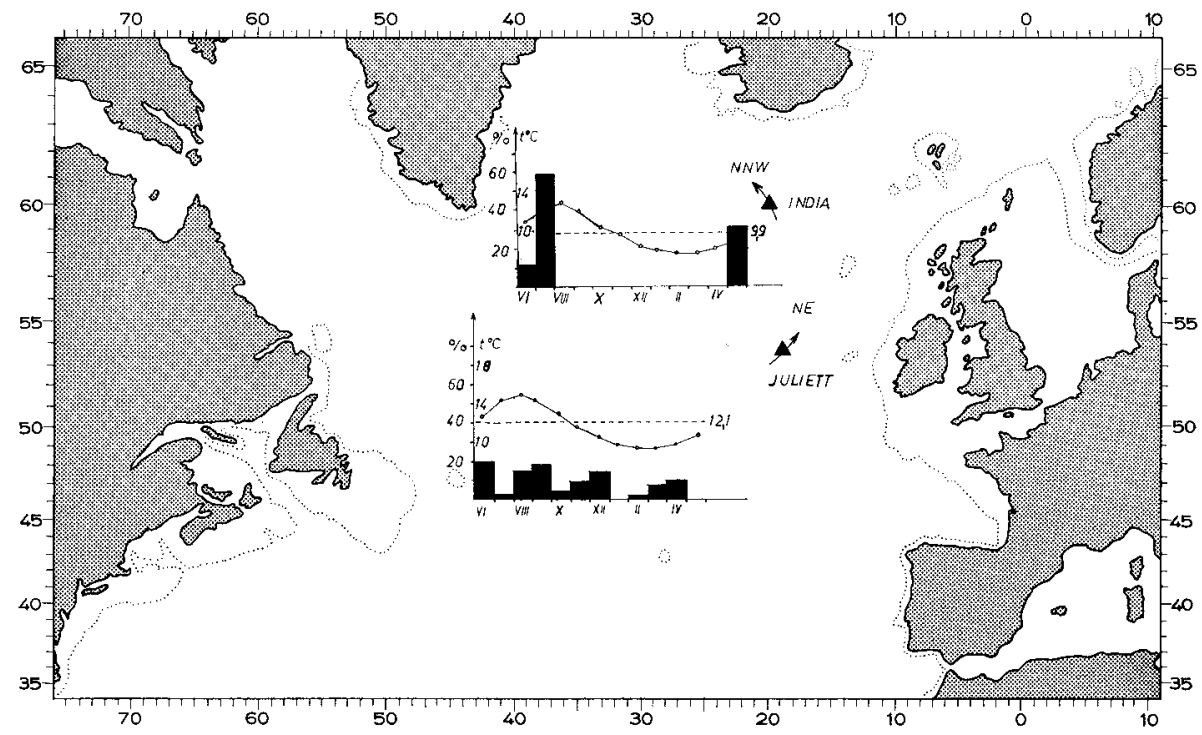

Abb. 6: Prozentuale Häufigkeit der Tintinnen bei den Wetterschiffstationen India und Juliett

sondern Gradienten scheinen wirksam zu sein. Nach CAMPBELL (1954) ist es offensichtlich, daß sich die Tintinnen in der lichtdurchfluteten Zone des Meeres aufhalten, wo das Angebot an Nahrung am größten ist.

Die jahreszeitliche Verbreitung von Tintinnen wurde an Hand von 63 Proben untersucht, die von den ozeanischen Wetterschiffstationen India $\left(\varphi=60^{\circ} \mathrm{N}, \lambda=\right.$ $\left.20^{\circ} \mathrm{W}\right)$ und Juliett $\left(\varphi=53^{\circ} 50^{\prime} \mathrm{N}, \lambda=18^{\circ} 40^{\prime} \mathrm{W}\right)$ stammen ${ }^{2}$. Die Proben wurden in den Jahren 1948/1949 mit einem Planktonnetz vertikal von 50 bis $0 \mathrm{~m}$ genommen. Das benutzte Netz ist von OSTENFELd \& JESPERSEN (1924) beschrieben worden. Es hat eine vordere Offnung von $1 / 5 \mathrm{~m}^{2}$ und die lichte Weite der Maschen beträgt $55 \mu$. Bei der Untersuchung der Fänge wurde mit einer Stempelpipette eine bestimmte Menge Plankton entnommen und bei einer Vergrößerung von $100 \times$ gezählt. Die formolfixierten Proben waren trotz ihres Alters in sehr gutem Zustand. Die gezählten Tintinnen wurden vermessen und das Plasmavolumen berechnet. Dabei wurde zugrunde gelegt, daß der Weichkörper die Hälfte des Volumens der Hülse ausfüllt.

Als Beispiel für den Jahresgang der Entwicklung von Tintinnen ist in Abbil-

${ }^{2}$ Dic Proben wurden mir freundlicherweise von Herrn J. CoRLetr, Fisheries Laboratory Lowestoft, zur Verfügung gestellt. 
dung 6 die prozentuale Häufigkeit der Tintinnen von April 1948 bis Mai 1949 bei den Stationen India und Juliett angegeben. Wetterstation India liegt zentral im verhältnismäßig kalten, nach NNW setzenden Irmingerstrom. Das Jahresmittel der Oberflächentemperatur betrug 1948 9,90 C; die Station Juliett liegt etwa 370 sm südlicher

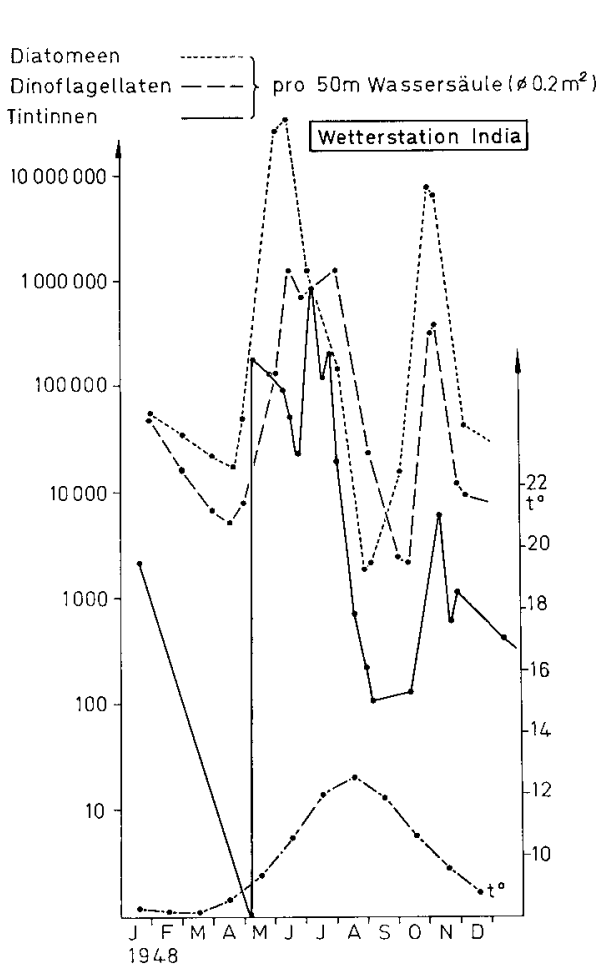

Abb. 7

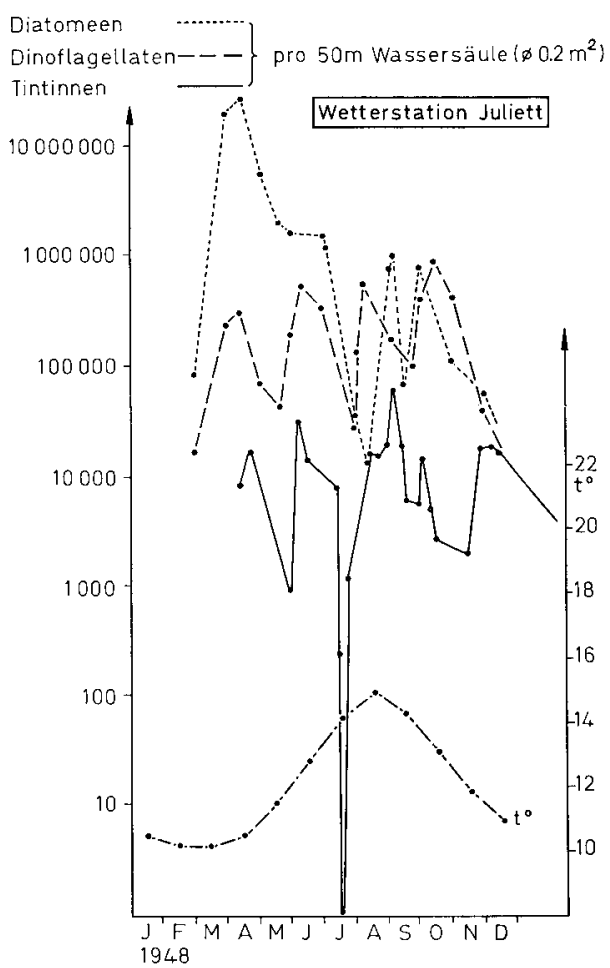

Abb. 8

Abb. 7: Jahreszeitliche Verteilung von Diatomeen und Dinoflagellaten (nach CoRLETT 1953) sowie Tintinnen im Jahre 1948 bei der Wetterschiffstation India

Abb. 8: Jahreszeitliche Verteilung von Diatomeen und Dinoflagellaten (nach CoRLETT 1953) sowie Tintinnen im Jahre 1948 bei der Wetterschiffstation Juliett

im warmen, nach NE setzenden Nordostatlantischen Strom. Hier wurde im gleichen Zeitraum an der Oberfläche eine mittlere Jahrestemperatur von $12,1^{\circ}$ errechnet. Sie war also 2,20 wärmer als bei Station India (Rodewald 1952).

Das Maximum der Tintinnenentwicklung bei Station India liegt eindeutig im Juli, von August bis März wurden nur sehr geringe Tintinnenmengen gezählt. Anders liegen die Verhältnisse bei Station Juliett. Hier sind das ganze Jahr über etwa gleiche Häufigkeiten beobachtet worden.

In den Abbildungen 7 und 8 sind die Individuenzahlen der Tintinnen für das Jahr 1948 angegeben, die mit der Häufigkeit von Diatomeen und Dinoflagellaten verglichen wurden (CORLETT 1953). Bei India ist die Hauptentwicklung der Tintinnen 
auf die Monate Mai bis Juli beschränkt. Maximal wurden etwa 4000000 Exemplare unter $1 \mathrm{~m}^{2}$ Oberfläche gezählt. Hauptsächlich vertreten sind Kaltwasserformen wie zum Beispiel Parafavella parumdentata, P. edentata und Ptychocylis minor. Die Hauptentwicklung an dieser Station fällt mit der Erwärmung des Wassers in diesem Meeresgebiet zusammen (April 8,6 $6^{\circ} \mathrm{C}$, Juli 12,00 C). Beim Maximum der Temperatur im August mit $12,6^{\circ} \mathrm{C}$ ist der Höhepunkt der Entwicklung bereits überschritten (nur 4000 Exemplare unter $1 \mathrm{~m}^{2}$ ). In die Zeit der Maximalentwicklung der Tintinnen fällt

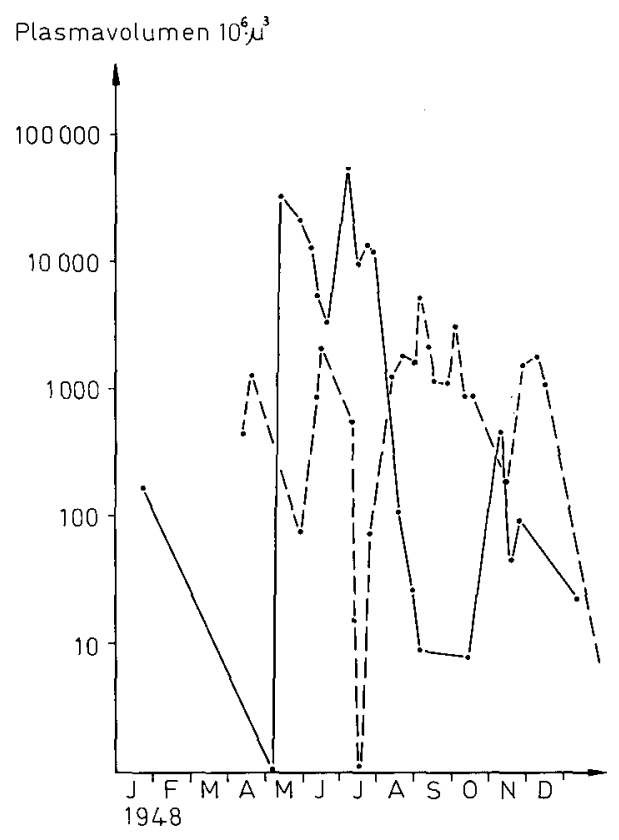

Abb. 9: Änderung des errechneten Plasmavolumens von Tintinnen im Verlauf des Jahres 1948 bei den Wetterschiffstationen India (ausgezogene Linie) und Juliett (gestrichelte Linie; Wassersäule $50 \mathrm{~m}$, Durchmesser des Netzes $0,2 \mathrm{~m}^{2}$ )

ebenfalls die Blüte der Diatomeen und Dinoflagellaten. Ebenso wie bei diesen beiden Phytoplanktongruppen, tritt bei den Tintinnen im November ein zweiter Entwicklungshöhepunkt ein, der jedoch mit 30000 Exemplaren unter $1 \mathrm{~m}^{2}$ Oberfläche erheblich geringer als das Sommermaximum ist.

Bei der südlichen Station Juliett ergibt sich ein anderes Bild. Die Individuenzahl der Tintinnen ist erheblich niedriger, die Zahl der Arten jedoch höher (vor allem Warmwasserarten wie Dictyocysta elegans und Eutintinnus fraknòi). Zu einem Maximum mit 61000 Exemplaren unter $1 \mathrm{~m}^{2}$ Oberfläche kam es Anfang September. Es treten mehrere Entwicklungsphasen ein, die ähnlich verlaufen wie bei den Dinoflagellaten.

$\mathrm{Da}$ die Individuenzahlen für produktionsbiologische Überlegungen ein schlechtes Maß sind - Größe der Exemplare und Arten können sehr verschieden sein - wurde für die Tintinnen das Plasmavolumen errechnet. 
In Abbildung 9 sind für Station India und Juliett die Plasmavolumina dargestellt, bezogen auf den Gesamtfang der Tintinnen. Die Werte auf der Ordinate sind in $10^{6} \cdot \mu^{3}$ angegeben. Der Verlauf dieser Plasmavolumenkurven ist ähnlich der Kurve der Gesamttintinnen. Das Maximum bei Station India tritt ebenfalls von Mai bis Juli mit $60000 \cdot 10^{6} \mu^{3}$ auf; das entspricht $300 \mathrm{~mm}^{3}$ unter $1 \mathrm{~m}^{2}$ Oberfläche. $\mathrm{Zu}$ einem zweiten Höhepunkt kommt es im November mit etwa $500 \cdot 10^{6} \mu^{3}$. Weitaus niedriger und gleichförmiger verläuft die Kurve der Station Juliett. Die meisten Werte bewegen sich um $1000 \cdot 10^{6} \mu^{3}$.

Quantitative Angaben über das Vorkommen von Tintinnen sind in der Literatur sehr spärlich vorhanden. Einige Daten sind in Tabelle 1 zusammengestellt worden.

Als begrenzende Faktoren für die Entwicklung der Tintinnen kommen vor allem Temperatur, Salzgehalt, Sauerstoff und Wasserstoffionenkonzentration in Frage. Nach Untersuchungen von Biernacka (1948), Gillbricht (1954) und Posta (1963) ist eindeutig, daß die Temperatur der wichtigste Faktor ist. BIERnAcka (1948) stellte in der Ostsee fest, daß bestimmte Tintinnopsis-Arten nur in einem sehr engen Temperaturbereich ihr Hauptvorkommen haben. Zu gleichen Ergebnissen kam Posta (1963) in Mittelmeer. Der Einfluß von Salzgehalt und Sauerstoffkonzentration tritt nach Ansicht von BIERnACKA weit zurück. Angaben über die Bedeutung der Wasserstoffionenkonzentration liegen noch nicht vor. Nach Kitching (1957) ist sie jedoch für viele Protozoen recht wichtig.

\section{DIE ROLLE DER TINTINNEN IN DER NAHRUNGSKETTE}

Um Aufschluß darüber zu erhalten, welchen Platz die Tintinnen in der Nahrungskette einnehmen, ist es erforderlich, ihre Nahrung sowie ihre Konsumenten kennenzulernen.

Die Nahrung der Tintinnen besteht aus Detritus, Bakterien, nackten Flagellaten, Coccolithophoriden, Peridineen, Diatomeen und Silicoflagellaten. Die Nahrungsteilchen werden im Peristomfeld gesammelt und mit Hilfe des "Stempels" - eine Sonderbildung bei dieser Ciliatengruppe - durch pumpenförmige Bewegungen durch den Zellmund in den Schlund transportiert.

Nach Angaben von ENTZ (1909) sind Tintinnen sehr gefräßig, und nach DADAY (1887) fressen sie sich sogar gegenseitig. Kofoid \& CAmpbell (1939) nehmen an, daß Tintinnen ihre Nahrung selektiv aufnehmen können; gewisse Arten fressen nur bestimmte Coccolithophoriden, auch wenn eine Vielzahl nebeneinander vorkommt. Im Zellinneren sind Tintinnen oft gelbgrün gefärbt. Die Färbung rührt sicherlich von Beuteobjekten her. CAMPBELL (1954) hält es jedoch für möglich, daß Tintinnen Zoochlorellen besitzen - ähnlich wie einige Paramaecium-Arten. Merkle (1909) ist der Ansicht, daß Tintinnen imstande sind, Gehäuse von Diatomeen und Peridineen aufzulösen und die gelöste Gehäusemasse zum Bau der eigenen Lorica wieder zu benutzen.

Lohmann (1908) fand im Plasma von Tintinnen der Kieler Bucht häufig kleine kugelige, halbverdaute und verfärbte rote und gelbe Zellen, die wohl sicher auf gefressene Rhodomonas und Chrysomonadinen zurückgeführt werden können. LOHMANN 


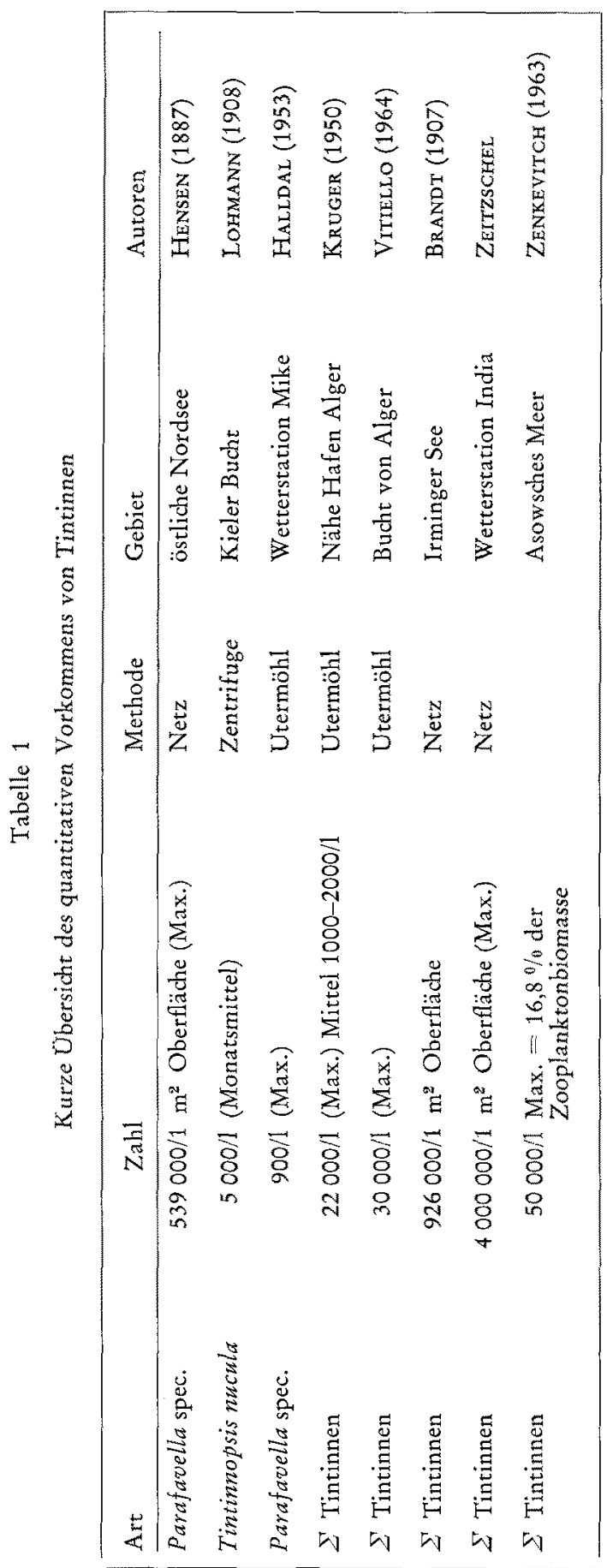


stellte fest: „Da die Tintinnen auf jeden Fall nur sehr kleine Organismen als Nahrung aufnehmen können, nahm ich an, daß sie als Vermittler zwischen diesen und Metazoen eine hervorragende Rolle spielen. Aber es scheint nach den vorliegenden Befunden, daß von den Metazoen selbst die Hauptmasse des pflanzlichen Mikroplanktons direkt gefressen wird und nur ein sehr unbedeutender Teil den Umweg durch die Tintinnen nimmt."

Dieser Annahme widersprechen, wenn auch für andere Meeresgebiete, die Angaben von Ponomareva $(1955,1962,1963)$ und eigene Untersuchungen (Zeitzschel 1966). Ponomareva untersuchte die Nahrung von Euphausiaceen im Nordpazifik und im Indischen Ozean. Sie fand, daß Euphausiaceen im Frühjahr und Sommer vor allem Phytoplankton fressen, während im Herbst Tintinnen und Foraminiferen den Hauptteil der Nahrung ausmachten. Ich untersuchte Euphausiaceen, die aus dem Gebiet nordwestlich von Schottland stammten. In einem einzigen Magen konnten bis zu 4000 Tintinnen gezählt werden. Als weitere Konsumenten sind Copepoden, Tunicaten und Fischlarven nachgewiesen, die zumindest zeitweise erhebliche Mengen Tintinnen fressen.

Die Tintinnen sind eines der ersten Glieder in der Nahrungskette und können daher bei produktionsbiologischen Betrachtungen von erheblicher Bedeutung sein. Man sollte ihnen deshalb in Zukunft bei derartigen Untersuchungen mehr Beachtung schenken. Durch ihre Kleinheit werden sie von Netzen mit Maschenweiten über $50 \mu$ nicht quantitativ gefangen. Untersuchungen von LoHmanN (1908) und Schwarz (1959) haben gezeigt, daß besonders von kleinen Tintinnenarten unter $40 \mu$ nur etwa $10 \%$ gefangen werden. Deshalb sollte man für quantitative Untersuchungen möglichst Wasserschöpfproben verwenden (UTERMöHL 1958) oder mit Netzfängen arbeiten, die mit sehr feiner Gaze $(<45 \mu)$ genommen wurden.

\section{ZUSAMMENFASSUNG}

1. Viele Tintinnenarten sind kosmopolitisch verbreitet. Gebiete des Weltmeeres mit ähnlichen Lebensbedingungen beherbergen gleiche Tintinnen. Sie leben in der lichtdurchfluteten Zone des Meeres, wo das Angebot an Nahrung am größten ist. Tägliche vertikale Wanderungen wurden nachgewiesen.

2. Die jahreszeitliche Verbreitung richtet sich nach der geographischen Breite. Bei der ozeanischen Wetterschiffstation India liegt das Maximum mit 4000000 Exemplaren unter $1 \mathrm{~m}^{2}$ im Mai bis Juli; das entspricht einer Biomasse von etwa $300 \mathrm{~mm}^{3}$.

3. Der begrenzende Faktor für die Entwicklung und Verbreitung von Tintinnen ist vor allem die Wassertemperatur. Der Einfluß von Salzgehalt und Sauerstoffsättigung ist offensichtlich weniger bedeutsam.

4. Die Nahrung der Tintinnen besteht aus Detritus, Bakterien, nackten Flagellaten, Coccolithophoriden, Peridineen, Diatomeen und Silicoflagellaten. Die Tintinnen selbst werden von Euphausiaceen, Copepoden, Tunicaten und Fischlarven gefressen.

5. Tintinnen bilden eines der ersten Glieder in der Nahrungskette. Man sollte in $\mathrm{Zu}$ kunft dieser interessanten Ciliatengruppe bei produktionsbiologischen Untersuchungen mehr Beachtung schenken. 


\section{ZITIERTE LITERATUR}

Brernacka, J., 1948. Tintinnoinea in der Bucht von Danzig und in den angrenzenden Gewässern. [Poln.] Biul. morsk. Inst. ryb. Gdyni 4, 73-91.

BRANDT, K., 1906. Die Tintinnodeen der Plankton-Expedition. Tafelerklärungen nebst kurzer Diagnose der neuen Arten. In: Ergebnisse der Plankton-Expedition der Humboldt-Stiftung. Hrsg. von V. Hensen. Lipsius \& Tischer, Kiel 3 (L. a.), 1-33.

- 1907. Die Tintinnodeen ... Systematischer Teil. In: Ergebnisse der Plankton-Expedition der Humboldt-Stifung ....3 (L. a.), 1-499.

Campbell, A. S., 1954. Protozoa (chiefly Radiolaria and Tintinnina). In: Treatise on invertebrate paleontology. Ed. by R. C. Moore. Geol. Soc. of America, New York; Univ. of Kansas pr., Lawrence, Pr D. Protista. 3, 1-195.

Claparède, E. \& Lachmann, J., 1858-59. Études sur les infusoires et les rhizopodes. Mém. Inst. natn. genev. 5 (3), 1-260; 6 (1), 262-482; 7 (1), 1-291.

CorletT, J., 1953. Net phytoplankton at ocean Weather Stations "I" and "J". J. Cons. perm. int. Explor. Mer. 19 (2), 178-190.

DaDAY, E., 1887. Monographie der Familie der Tintinnideen. Mitt. zool. Stn Neapel 7, 473-591.

ENTZ, G., Jr., 1909. Studien über Organisation und Biologie der Tintinnideen. Arch. Protistenk. $15,93-226$.

Fauré-Fremiet, E., 1924. Contribution à la connaissance des infusoires planktoniques. Bull. biol. Fr. Belg. (Suppl.) 6, 82-119.

Grlibricht, M., 1954. Das Verhalten von Zooplankton - vorzugsweise von Tintinnopsis beroidea ENTz - gegenüber thermohalinen Sprungschichten. Kurze Mitt. Inst. FischBiol. Univ. Hamb. 5, 32-44.

GökE, G., 1963. Meeresprotozoen (Foraminiferen, Radiolarien, Tintinninen). Franckh, Stuttgart, 75 pp.

Halldal, P., 1953. Phytoplankton investigations from Weather Ship $M$ in the Norwegian sea 1948-49. Hvalråd. Skr. 38, 1-91.

Hensen, V., 1887. Über die Bestimmung des Planktons oder des im Meere treibenden Materials an Pflanzen und Tieren. Ber. dt. wiss. Kommn Meeresforsch. 5, 1-108.

Jørgensen, E., 1924. Mediterranean Tintinnidae. Rep. Dan. oceanogr. Exped. Mediterr. 2, (Biology, J 3), 1-110.

- 1927. Ciliata: Tintinnidae. In: Die Tierwelt der Nord- und Ostsee. Begr. von J. G. Grimpe \& A. E. Wagler. Geest \& Portig, Leipzig, 1 (2c1.2.), 1-28.

Kitching, J. A., 1957. Some factors in the life of free-living protozoa. In: Microbial ecology. Ed. by R. E. O. Williams \& C. C. Spicer. Univ. pr., Cambridge, 388 pp. (Symp. Soc. gen. Microbiol. 7, 259-286).

Koford, C. A. \& CAMpbell, A. S., 1929. A conspectus of the marine and fresh-water ciliata belonging to the suborder Tintinnoinea, with descriptions of new species principally from the Agassiz Expedition to the Eastern Tropical Pacific 1904-1905. Univ. Calif. Publs Zool. 34, 1-403.

- - 1939. Reports on the scientific results of the expedition to the eastern tropical Pacific in charge of Alexander Agassiz, by the U. S. Fish Commission steamer "Albatross" from October, 1904, to March, 1905. 37: The Ciliata: The Tintinnoinea. Bull. Mus. comp. Zool. Harr. 84, 1-473.

KRUGER, D., 1950. Variations quantitatives des protistes marins au voisinage du port d'Alger durant l'hiver 1949-50. Bull. Inst. océanogr. Monaco (978), 1-19.

Lohmann, H., 1908. Untersuchungen zur Feststellung des vollständigen Gehaltes des Meeres an Plankton. Wiss. Meeresunters, (Abt. Kiel) 10, 129-370.

Merkie, H., 1909. Untersuchungen an Tintinnodeen der Ost- und Nordsee. Wiss, Meeresunters. (Abt. Kiel) 11, 139-186.

MüLler, O. F., 1776. Zoologiae Danicae Prodromus seu animalium Daniae et Norvegiae indigenarum characteres, nomina et synonyma imprimis popularium. Havniae, Typ. Hallagerüs, $274 \mathrm{pp}$. 
Ostenfeld, C. H. \& Jespersen, P., 1924. Standard net for plankton collections. Publs Circonst. Cons. perm. int. Explor. Mer 84, 1-16.

Ponomareva, L. A., 1955. The feeding and distribution of euphausiids in the sea of Japan. [Russ.] Zool. $\mathrm{Zh}$. 34, 85-97.

- 1963. The Euphausiids of the North Pacific, their distribution and ecology of common species. [Russ.] Akad. Nauk SSSR, Moskva, 141 pp.

- Naumov, A. G. \& Zernova, V. V., 1962. Food composition of some Euphausiid species of the Indian Ocean. [Russ.] Trudy Inst. Okeanol. 58, 163-166.

Posta, A., 1963. Relation entre l'érolution de quelques Tintinnides de la rade de Villefranche et la température de l'eau. Cah. Biol. mar. 4 (2), 201-210.

Rodewald, M., 1952. Wassertemperaturen im Nordatlantik und Nordmeer 1948-1951. Fischereiwelt 3, 43-44.

- Normalwerte und Abweichungen der Meerestemperaturen bei den nordatlantischen Wetterschiffen. Dt. bydrogr. Z. 5, 131-140.

SCHWARZ, S., 1959. Vergleichende Studien an tierischem Netz- und Vollplankton aus Brackwassergebieten der Ostsee. Z. Fisch. 8, 351-370.

SCHWEYER, A. W., 1909. Zur Kenntnis des Tintinnodeenweichkörpers, nebst einleitenden Worten über die Hülsenstruktur und die Hülsenbildung. Arch. Protistenk. 18, 134-189.

UtermöHL, H., 1958. Zur Vervollkommnung der quantitativen Phytoplankton-Methodik. Mitt. int. Verein. theor. angew. Limnol. 9, 1-38.

Vitrello, P., 1964. Contribution à l'étude des Tintinnides de la baie d'Alger. Pelagos (Bull. Inst. océanogr. Alger) 2 (2), 5-42.

Zeitzschel, B., 1966. Die Verbreitung der Tintinnen im Nordatlantik. Veröff. Inst. Meeresforsch. Bremerh. (Sonderbd) 2, 293-300.

ZeNKevitch, L., 1963. Biology of the seas of the U.S.S.R. Transl. from the Russ. Allen \& Unwin, London, $955 \mathrm{pp}$.

\section{Diskussion im Anscbluß an den Vortrag ZeITZSCHeL}

KINNE: Was fressen Tintinnen? Wer frißt Tintinnen? Im Hinblick auf die starken jährlichen Abundanzschwankungen - welche Sie in Ihrem Vortrag primär auf die Wirkung abiotischer Faktoren (Temperatur, Salzgehalt, Sauerstoff) zurückführen - scheint mir die Beantwortung dieser beiden Fragen von Wichtigkeit. Vermutlich spielen neben abiotischen Faktoren biotische Korrelationen, vor allem innerhalb der Nahrungskette, eine entscheidende Rolle. Hier müssen experimentell-ökologische Untersuchungen im Laboratorium die im Meer gewonnenen $\mathrm{Be}$ funde ergänzen. Allerdings kann ich mir vorstellen, daß es durchaus nicht einfach ist, Tintinnen zu kultivieren; liegen hier schon Erfahrungen vor?

ZerTzsCheL: Über die Beziehung zu anderen Organismen liegen bisher keine Untersuchungen vor. Ich nehme an, daß sich Tintinnen vor allem von sehr kleinen Organismen - etwa Bakterien und nackten Flagellaten - ernähren. Derartige Organismen kann man nur schwer im Zellinnern der Tintinnen nachweisen. Es ist wahrscheinlich, daß nach einer Hauptentwicklung von Tintinnen deren Konsumenten ebenfalls eine maximale Entwicklung durchmachen, was zur Folge haben würde, daß dann die Tintinnen in ihrer Zahl stark reduziert werden. Es sind also vermutlich nicht nur physikalische sondern auch biologische Faktoren von Bedeutung. Im einzelnen wird man diese Fragen nur durch Kulturexperimente klären können. Bisher sind jedoch fast alle Kulturversuche fehlgeschlagen. Ich beabsichtige, in Zukunft diese Arbeiten zu intensivieren und hoffe, bald nähere Angaben zu diesen Fragen machen zu können.

LAKKIS: Dans votre séjour au Laboratoire d'Océanographique d'Edinburg est-ce que vous aviez constaté que les échantillons sont très pauvres en Tintinnides? Est-ce que ceci est dû à l'incompétence du "Continuous Plankton Recorder» utilisé pour le prélèvement des échantillons de toutes les régions de l'Atlantique du Nord, ou bien à la répartition en profondeur des Tintinnides? (L'apparail échantillone à une profondeur de $10 \mathrm{~m}$ seulement.) 
ZeITZsChel: Die im Hardy Plankton Recorder benutzte Gaze hat eine Maschenweite von etwa $240 \mu$. Bei Experimenten in den Gewässern um Millport und in der Nordsee haben wir festgestellt, daß vom Phytoplankton mit dieser Gaze nur etwa 1 bis $3 \%$ gefangen werden. Ein ähnliches Fangergebnis gilt für Tintinnen. Laboratoriumsexperimente mit Parafavella gigantea $(500 \mu)$ haben ergeben, daß von dieser großen Form nur $3 \%$ von der Recordergaze zurückgehalten werden.

Remane: Als Planktonlaie möchte ich mir eine Frage erlauben. Wie kommt das doppelte Maximum im Jahresablauf zustande? Es betriff nicht nur Diatomeen, Peridineen und Tintinnen, sondern auch Rotatorien. Es kommt im Nordatlantik ebenso vor wie in der Kieler Bucht. In manchen Jahren fällt ein Maximum aus, meist das zweite, gelegentlich das erste, wenigstens bei Rotatorien. Leicht verstehbar ist das erste Maximum, wie steht es mit dem zweiten?

ZeItzschel: Für die Phytoplanktonblüte im Frühjahr ist neben dem Vorhandensein an Nährstoffen die Stabilisation des Wassers und das Lichtangebot von großer Bedeutung. In unseren Breiten ergeben sich meist 2 Maxima. Das erste ist beendet, wenn einer der begrenzenden Nährstoffe - Nitrate oder Phosphate - verbraucht ist. Durch Mineralisation wird jedoch im Spätsommer ein Teil dieser Nährstoffe wieder frei; dieser Umstand erlaubt eine zweite Entwicklungsphase.

MARGALEF: I want to emphasize the fact, that nets are not appropriate for quantitative collection of Tintinnids; they give too small numbers. Study of water samples with UTERMÖHL's microscope gives more fair estimates, also because naked forms outnumber shelled species that are the only ones collected by net tows. I refer to dates published in Rapports et Procès Verbaux du C.I.E.S.M. (1962).

ZeITzschel: Ich stimme Ihnen bei, daß für quantitative Bestimmungen von Tintinnen UTERMÖHL-Proben besser sind als Netzfänge. Für die Analyse der jahreszeitlichen Abundanzschwankungen der Tintinnen im offenen Nordatlantik standen mir jedoch nur die erwähnten Netzproben von den Wetterschiffstationen "Juliett" und "India“ zur Verfügung. Bei der Bearbeitung von UTERmöHL-Proben, besonders aus oligotrophen Meeresteilen, muß man jedoch darauf achten, daß die Wasserproben für die Zählungen mit dem umgekehrten Mikroskop mindestens 11 betragen sollten, um die Zählergebnisse statistisch absichern zu können. 